\section{AB1283 WHEN SHOULD WE EVALUATE CYTOMEGALOVIRUS INFECTION IN IMMUNOSUPPRESSED PATIENTS WITH CONNECTIVE TISSUE DISEASES?}

Yoichi Nakayama ${ }^{1}$, Ryuichi Sada ${ }^{1}$, Hirofumi Miyake ${ }^{1}$, Hiroyuki Akebo ${ }^{1}$, Yukio Tsugihashi' ${ }^{2}{ }^{1}$ Tenri Hospital, Department of General Internal Medicine, Tenri, Japan; ${ }^{2}$ Tenri Healthcare University, Center for Healthcare Education and Research, Tenri, Japan

Background: Cytomegalovirus (CMV) infection is an opportunistic and often problematic infection in patients with connective tissue diseases (CTD patients) who undergoes immunosuppressive therapy. However, in immunosuppressed CTD patients, CMV infection tends to be overdiagnosed, because it manifests with various symptoms and shows organ involvement. Little is known about when to suspect CMV infection and when to conduct $\mathrm{CMV}$ pp65 antigenemia assay in immunosuppressed CTD patients.

Objectives: To investigate the characteristics of patients who underwent CMV antigenemia assay and identify contributing factors of CMV infection in immunosuppressed CTD patients in a single center of Japan.

Methods: Medical records of hospitalized CTD patients who underwent CMV antigenemia assays between April 2015 and July 2018 were retrospectively reviewed. Patients were divided into groups with/without CMV antigenemia. Clinical features, basal immunosuppressive therapies, and laboratory data were analyzed.

Results: Overall, 108 patients were enrolled into the study; of these, the positive (group A) and negative (group B) CMV antigenemia groups had 49 and 59 patients, respectively. The underlying CTDs included systemic lupus erythematosus $(n=27)$, anti-neutrophil cytoplasmic antibody-associated vasculitis $(n=26)$, rheumatoid arthritis $(n=22)$, polymyositis/dermatomyositis $(n=13)$, and others $(n=20)$. Glucocorticoid was used in $101 \quad(93.5 \%)$ patients, and mean prednisolone dose was $27.1 \pm 18.3 \mathrm{mg} /$ day. Pulse glucocorticoid therapy and intravenous cyclophosphamide (IVCY) therapy was administered in $14(13.0 \%)$ and $22(20.4 \%)$ patients, respectively.

Group A patients had a significantly higher rate of Charlson Comorbidity Index $(\mathrm{CCl}) \geq 3(69.4 \%$ vs. $30.5 \%, \quad \mathrm{p}<0.001)$. Mean prednisolone dose was significantly higher in Group A $(32.9 \pm 15.5 \mathrm{mg} /$ day vs. $22.3 \pm 19.0 \mathrm{mg} /$ day, $\mathrm{p}=0.001)$. Pulse glucocorticoid therapy $(26.5 \%$ vs. $1.7 \%, \mathrm{p}<0.001)$ and IVCY $(36.7 \%$ vs. $6.8 \%, p<0.001)$ were administered significantly more often to Group A patients. Myelosuppression was seen significantly more often in Group A $(61.2 \%$ vs $39.0 \%, p=0.035)$. Laboratory data, including serum lymphocyte counts $(1016 \pm 1136 / \mu$ l vs. $1218 \pm 718 / \mu l)$, hemoglobin level $(10.5 \pm 2.6 \mathrm{~g} / \mathrm{dL}$ vs. $11.5 \pm 2.1 \mathrm{~g} / \mathrm{dL})$, and platelet counts $(14.5 \pm 9.7$ $\times 10^{6} / \mu \mathrm{l}$ vs. $\left.24.1 \pm 12.9 \times 10^{6} / \mu \mathrm{l}\right)$ were significantly lower in Group A $(\mathrm{p}<0.05)$.

Multivariate logistic regression analysis revealed $\mathrm{CCl} \geq 3$ (odds ratio (OR) $=3.21,95 \% \mathrm{Cl} 1.13-9.16)$ and IVCY therapy $(\mathrm{OR}=8.89,95 \% \mathrm{Cl} 1.99$ 39.7) as independent risk factors of CMV antigenemia. The result suggested that pulse glucocorticoid therapy could relate to CMV antigenemia (OR $=9.07,95 \% \mathrm{Cl}$ 0.96-85.7).

Based on the prediction criteria for CMV antigenemia defined as the existence of one or more contributing factors including $\mathrm{CCl} \geq 3$, pulse glucocorticoid therapy, IVCY, and myelosuppression; sensitivity and specificity were $89.8 \%(95 \% \mathrm{Cl} 81.3-98.3)$ and $44.1 \%(95 \% \mathrm{Cl} 31.4-56.8)$, respectively. With contributing factors limited to $\mathrm{CCl} \geq 3$ and IVCY, sensitivity and specificity became $75.5 \%(95 \% \mathrm{Cl} 63.5-87.5)$ and $64.4 \%(95 \% \mathrm{Cl} 52.2-$ 76.6), respectively.

Conclusion: Having multiple comorbidities and being on intensive immunosuppressive therapy, such as pulse glucocorticoid therapy and IVCY, were related to CMV antigenemia in CTD patients. The prediction criteria, including $\mathrm{CCl} \geq 3$, pulse glucocorticoid therapy, IVCY, and myelosuppression are helpful to clinically rule out CMV infection.

Disclosure of Interests: None declared

DOI: 10.1136/annrheumdis-2019-eular.4681

\section{AB1284 THE PATIENT EXPERIENCE: A PROCESS EVALUATION OF A PILOT PRAGMATIC USING REMOTE MONITORING OF SYMPTOMS}

Alexis Ogdie ${ }^{1}$, Michael George ${ }^{1}$, Kathleen Bush ${ }^{1}$, Mitesh Patel ${ }^{1}$, W. Benjamin Nowell ${ }^{2}$, Joshua Baker'. ' ${ }^{1}$ University of Pennsylvania, Philadelphia, PA, United States of America; ${ }^{2}$ Global Healthy Living Foundation, Upper Nyack, NY, United States of America

Background: Traditional randomized controlled trials (RCTs) are important for testing drug efficacy but this study design is burdensome for patients (pts) and clinicians. Pragmatic trials addressing real world comparative effectivness are equally important but challenging to conduct. We piloted a trial platform aspiring to optimize the patient experience and minimize patient and physician burden. The trial (NCT02912221) tests an incentive strategy informed by behavioral economics to increase physial activity in pts with rheumatoid arthritis (RA) and psoriatic arthritis (PsA).

Objectives: To understand patient experiences using mobile applications (apps) and wearable activity devices (WAD) in a pilot pragmatic trial Methods: Pts had RA or PsA with active disease defined by a Routine Assessment of Patient Index Data (RAPID3) score>3 (range 0-30). After screening and informed consent, pts received a WAD (FitBit ${ }^{\mathrm{TM}}$ ) and were assisted in setting up the device and an ArthritisPower account, a research registry where pts track patient-reported outcomes (PRO). A web-based platform, WayToHealth, was used to collect WAD data and deliver incentives. After a two-week baseline period, pts selected a daily step goal and were randomized to a financial loss-aversion incentive arm or control. Pts in both arms received weekly text messages reporting the number of days the step goal was met. In-person assessments were conducted at baseline and 14 weeks; pts completed weekly PROs (RAPID3, PROMIS Fatigue, PROMIS Sleep Disturbance and adverse event assessment) via mobile app or a web link. At 14 weeks, pts underwent a semi-structured interview to assess the patient experience in the trial. Content analysis was used to evaluate the responses

Results: To date, 27 pts completed the 14-week follow-up interview. Mean age was 48 (SD 14), 85\% were women, 17 (63\%) had PsA and $10(37 \%)$ had RA. Mean disease duration was 9 years, mean swollen joint count (0-66) was 6.2 (SD 5.6) and tender joint count (0-68) was 8.1 (SD 9.1). The mean RAPID3 was 10.3 (SD 4.6). Overall pts enjoyed participation in the study and provided useful feedback for improvement (Table). Weekly PRO capture was acceptable to most but PROs need to be streamlined to remove repetitive questions. Pts frequently set goals they were not able to achieve and suggested the ability to change their goal in future studies

Conclusion: In this pilot pragmatic trial, only two in-office visits were conducted and all other data captured remotely. Pts enjoyed the experience and found the digital platform easy to use. Such trial designs will become increasingly important in conducting real-world comparative effectiveness and adjunct therapy trials

\section{Table: Lessons learned from patient perspective in pragmatic trial}

\section{Trial Format}

Pt enjoyed tracking progress

Weekly surveys were ok; 2 pts recommended EOW:

Weekly check ins kept pts accountable

Most pts completed surveys via a text link (over App or web portal)

Time to complete surveys was 5 min

Pt requested a text box to record reasons for not feeling well (e.g., injury, illness)

PROs

Repetitive questions in the PROMIS measures annoyed pts

$\mathrm{MDHAQ}$ questions were not always relevant

Tracking sleep helped understand level of fatigue

Emotional wellbeing questions were appreciated

Desire to track individual PRO goals

Wearable Activity Device

Served as a reminder to move

Annoyed by need to charge

Creating teams and self-competition was motivating and fun

Finger arthritis directed type of bracelet

Some exercise not captured (e.g., swimming, biking)

Physical activity

Goals frequently set too high; allow for a goal change

Weekly goals may be better than daily goals

Weather influenced physical activity; being accountable encouraged exercise indoors

Overexertion sometimes lead to pain and stiffness

Some reported knee/foot pain with increasing steps

Disclosure of Interests: Alexis Ogdie Grant/research support from: (To my university) Novartis, Pfizer, Grant/research support from: Novartis, Pfizer, Grant/research support from: Novartis, Pfizer, Grant/research support from: Novartis, Pfizer, Consultant for: AbbVie, Bristol-Myers Squibb, Celgene, Corrona, Eli Lilly and Company, Novartis, Pfizer, and Takeda, Consultant for: AbbVie, Amgen, Bristol-Myers Squibb, Celgene, Corrona, Eli Lilly, Novartis, Pfizer Inc, Takeda, Consultant for: Abbvie, Amgen, BMS, Celgene, Corrona, Lilly, Novartis, Pfizer, Takeda, Consultant for: Abbvie Amgen, BMS, Celgene, Corrona, Lilly, Novartis, Pfizer, Takeda, Michael George Grant/research support from: BMS, Consultant for: Abbvie, Kathleen Bush: None declared, Mitesh Patel: None declared, W. Benjamin Nowell: None declared, Joshua Baker: None declared DOI: 10.1136/annrheumdis-2019-eular.4866 